\title{
Úlcera rectal solitaria como simulador de cáncer colorrectal y enfermedad de Crohn: reporte de caso
}

\section{Solitary rectal ulcer simulating colorectal cancer and Crohn's disease: Case report}

\author{
Rómulo Vargas-Rubio, ${ }^{1}$ (c) Óscar Messa, ${ }^{2}$ (1) Karen Álvarez, ${ }^{3}$ (D) Diana Meza-Madrid. ${ }^{*}$ (c)
}

\author{
Gacceso abierto \\ Citación: \\ Vargas-Rubio R, Messa 0, Álvarez K, \\ Meza-Madrid D. Úlcera rectal solitaria como \\ simulador de cáncer colorrectal y enfermedad \\ de Crohn: reporte de caso. Rev Colomb \\ Gastroenterol. 2021;36(2):280-283. https://doi. \\ org/10.22516/25007440.660 \\ 1 Especialista en gastroenterología y endoscopia \\ digestiva. Jefe de Unidad gastroenterología del \\ Hospital Universitario San Ignacio. Pontificia \\ Universidad Javeriana, Bogotá, Colombia. \\ 2 Patólogo oncólogo. Hospital Universitario San \\ Ignacio. Bogotá, Colombia. \\ ${ }^{3}$ Residente de medicina interna, Pontificia \\ Universidad Javeriana. Hospital Universitario \\ San Ignacio, Bogotá Colombia. \\ ${ }^{4}$ Residente de gastroenterología. Pontificia \\ Universidad Javeriana, Hospital Universitario \\ San Ignacio. Bogotá, Colombia. \\ *Correspondencia: Diana Meza-Madrid. \\ pav3394@gmail.com \\ Fecha recibido: $\quad$ 09/09/20 \\ Fecha aceptado: 19/01/21 \\ (c) () $\Theta$
}

\begin{abstract}
Resumen
La úlcera rectal solitaria es una patología poco frecuente de naturaleza benigna que debe diferenciarse de otras entidades en su presentación inicial, con mecanismos fisiopatológicos definidos y con sintomatología variada. Se presenta el caso de una paciente de 31 años sin antecedentes de importancia, que consultó a nuestra institución por un cuadro de 3 años de sangrado rectal asociado con episodios de diarrea, manejada con múltiples diagnósticos.
\end{abstract}

\section{Palabras clave}

Enfermedades del recto, hemorragia gastrointestinal, enfermedades del colon.

\begin{abstract}
Solitary rectal ulcer is a rare condition of benign nature that must be differentiated from other disorders with defined pathophysiological mechanisms and varied symptoms. The following is the case of a 31-year-old female patient with no relevant history, who consulted our institution due to symptoms of rectal bleeding associated with episodes of diarrhea for three years, who received multiple diagnoses.
\end{abstract}

Keywords

Rectal diseases; Gastrointestinal bleeding; Colonic diseases.

\section{INTRODUCCIÓN}

El síndrome de úlcera rectal solitaria es una enfermedad benigna, poco frecuente, que presenta una variedad de síntomas, hallazgos clínicos y anomalías histopatológicas que la identifican. Fue descrita por primera vez por Cruveihier en 1829, cuando informó 4 casos de úlceras rectales (1). Sin embargo, el término úlcera rectal solitaria fue utilizado como tal por Lloyd-Davis hasta fines de la década de 1930 (2).

La incidencia es incierta, pero se estima que es de 1 en 100000 individuos por año, y se considera una entidad subdiagnosticada. Se confunde con otras patologías como la enfermedad inflamatoria intestinal (EII) y lesiones neoplásicas; ocurre con mayor frecuencia en la tercera década 
de la vida en hombres y cuarta década en mujeres, con un pequeño predominio en mujeres (3).

\section{DESCRIPCIÓN DEL CASO CLÍNICO}

Se trata de una paciente femenina de 31 años, que inició un cuadro clínico en marzo del 2016 en el que presentaba dolor abdominal tipo cólico y distensión abdominal asociado con rectorragia de cantidad abundante en múltiples episodios.

Se realizó la colonoscopia en mayo de 2016 y se identificó en la pared anterior del recto una irregularidad de la mucosa de $5 \mathrm{~cm}$ de diámetro sobre una base eritematosa, con engrosamiento de pliegues y friabilidad. El resultado histopatológico reportó una lesión tumoral maligna constituida por adenocarcinoma moderadamente diferenciado. Se remitió a oncología para la resección de la lesión tumoral; sin embargo, se consideró repetir la rectosigmoidoscopia. En el nuevo estudio histopatológico no se identificó malignidad. Debido a este nuevo hallazgo no se realizó el manejo oncológico. Después de 2 meses asintomática, reinició la rectorragia, por lo que se realizó la endoscopia nuevamente (Figura 1). Se observó una úlcera a $6 \mathrm{~cm}$ del reborde anal, histopatológicamente con cambios reactivos de las criptas y fibrosis sin cambios de malignidad. Recibió tratamiento sintomático y presentó remisión durante varios meses. En marzo de 2017 (Figura 2) se realizó una endoscopia por el reinicio de los síntomas y se identificó una úlcera rectal de $6 \mathrm{~cm}$ en el diámetro transversal, cubierta por fibrina con bordes engrosados y duros con resultado de histopatología que informó colitis crónica.
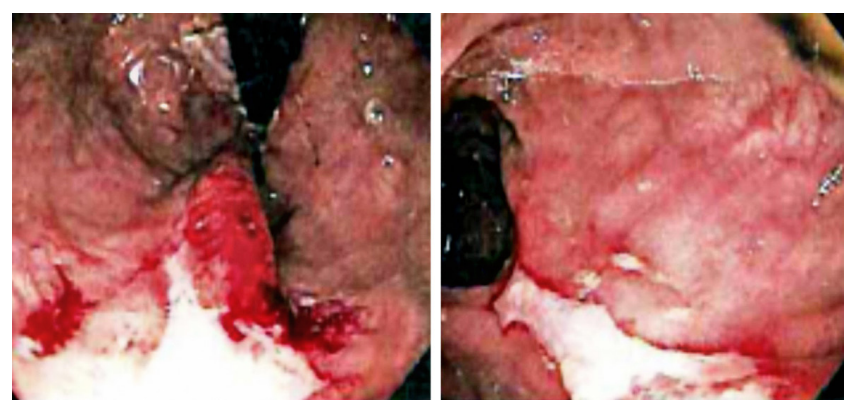

Figura 1. Colonoscopia de septiembre de 2016. Se observan a $6 \mathrm{~cm}$ del reborde anal una lesión ulcerada, bordes definidos cubiertos por fibrina sin sangrado de $3 \times 1 \mathrm{~cm}$ en el resultado histológico con mucosa rectal con cambios reactivos de las criptas, fibrosis y congestión vascular en la lámina propia sin cambios de malignidad.

Durante 2019 se realizó un control endoscópico y se observó a $5 \mathrm{~cm}$ del canal anal una úlcera de $3 \times 2 \mathrm{~cm}$ cubierta de fibrina. La biopsia reportó una úlcera crónica con cambios reactivos y fibrosis. Se diagnosticó con enfermedad de Crohn, y fue tratada únicamente con ciclos de prednisolona, con los cuales mejoraron los episodios de rectorragia.

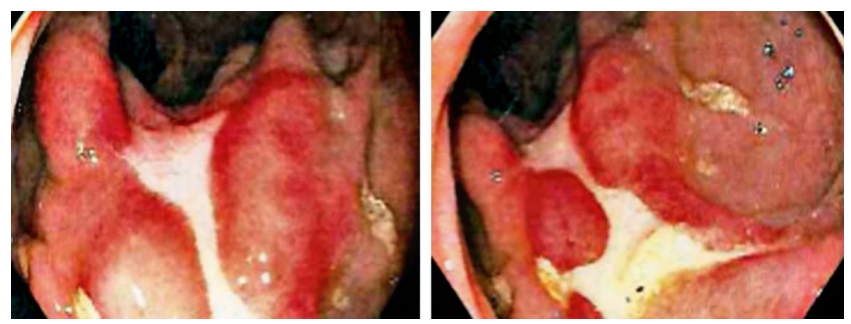

Figura 2. Colonoscopia de marzo de 2017. Se identificó una úlcera rectal de $6 \mathrm{~cm}$ transversal cubierta por fibrina y bordes engrosados y duros; el resto del estudio salió normal hasta el íleo distal. Con la biopsia de la úlcera se informó colitis crónica.

Fue evaluada por primera vez en nuestra institución en agosto de 2019 luego de 11 días de rectorragia con 10-12 episodios diarios, dolor abdominal de alta intensidad y fiebre. En el examen físico se encontró hemodinámicamente estable, con signos de deshidratación. Debido a los antecedentes se decidió iniciar el manejo como EII con un nuevo ciclo de prednisolona. Se solicitó la revisión de estudios de patología previos, realizados en mayo de 2016, y se realizó una ileocolonoscopia (Figura 3).

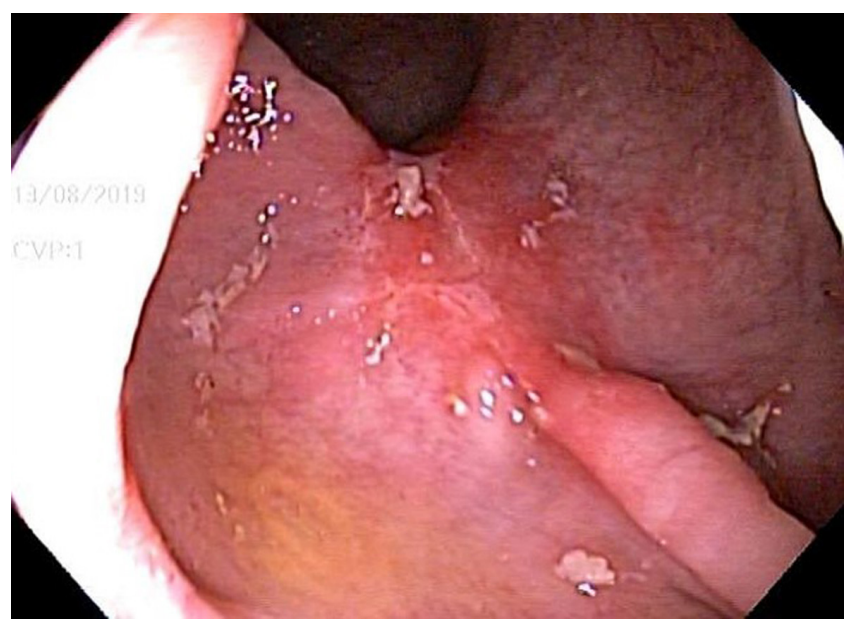

Figura 3. Rectosigmoidoscopia de agosto de 2019. Se observa desde la línea pectínea hasta la válvula de Houston inferior una alteración del patrón estructural con pérdida del patrón vascular, mucosa eritematosa, edema y nodularidad, leve friabilidad con retracción de la mucosa y cicatrización a nivel distal.

En la ileocolonoscopia se identificó la presencia de úlcera rectal solitaria, sin lesiones en la mucosa ileal. Las muestras de histopatología de mayo de 2016 fueron evaluadas por patólogos institucionales, en las que se encontró mucosa con artificios tisulares con presencia focal de grupos celulares con atipia citológica y arquitectónica (Figura 4), por lo que se planteó inicialmente adenocarcinoma frente a cambios reactivos inflamatorios. Sin embargo, en los cortes de inmunohistoquímica no se encontró representada la lesión. 
En las nuevas muestras obtenidas de forma institucional se encontró mucosa hiperplásica sin displasia y fragmento de úlcera sin lesiones tumorales (Figura 5); no se observó ningún cambio arquitectónico, microrganismos, granulomas ni un claro reemplazo fibromuscular de la lámina propia.

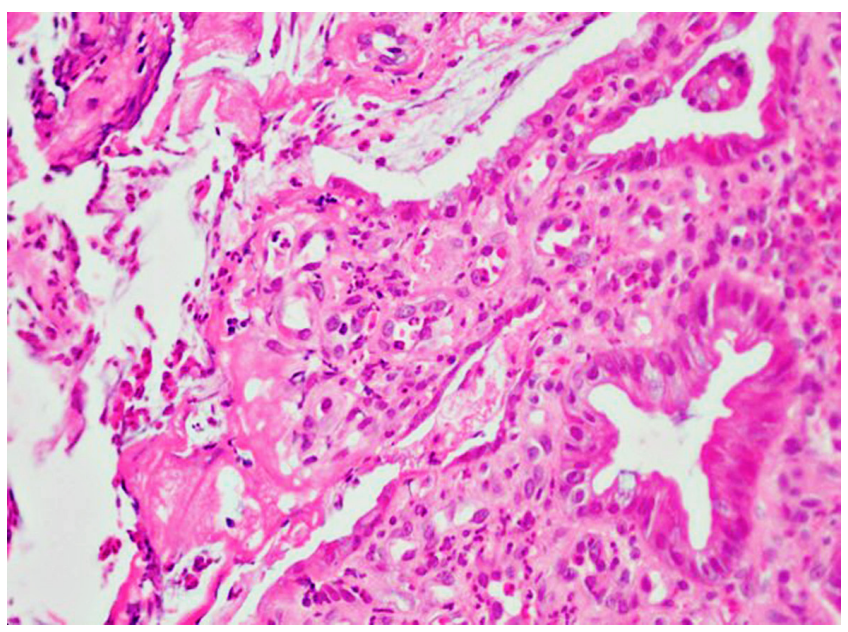

Figura 4. Reemplazo fibroinflamatorio de la lámina propia. Tinción de hematoxilina-eosina ( $\mathrm{H}$ y E): $10 \mathrm{X}$.

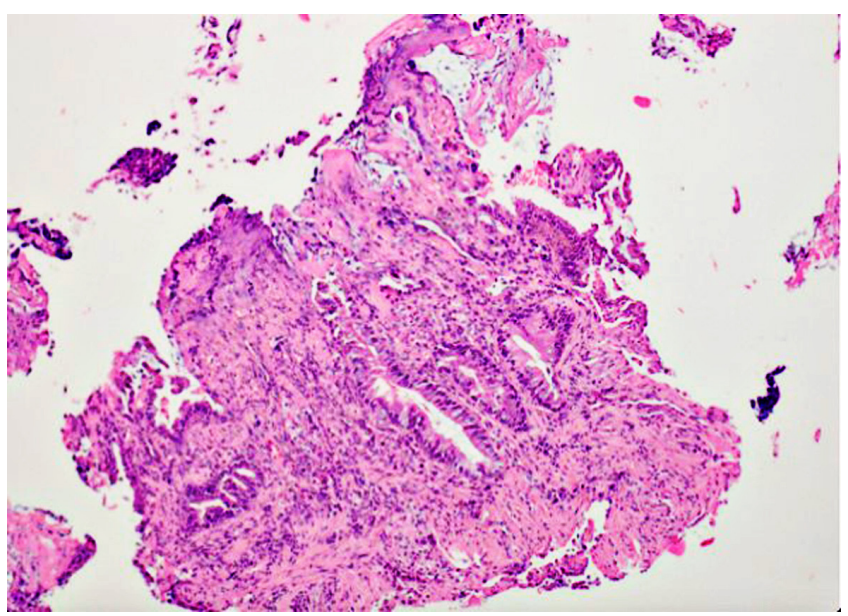

Figura 5. Mucosa con ulceración superficial con atípicas epiteliales reactivas. H y E: $40 \mathrm{X}$.

Después de la discusión del caso en la junta multidisciplinaria, se ampliaron los estudios con defecografía, que resultó altamente sugestiva de intususcepción rectal como causa de la lesión ulcerada persistente; se concluyó que se trata de un síndrome de úlcera rectal solitaria.

\section{DISCUSIÓN}

La úlcera rectal solitaria es una entidad poco frecuente. Su abordaje puede representar un reto dada su similitud con otras entidades. En cuanto a la dificultad diagnóstica, cabe resaltar que es fundamental una historia dirigida y evolutiva en el tiempo, con claros detalles de factores de riesgo y antecedentes. En este caso, al reinterrogar a la paciente se pudieron constatar síntomas de constipación y de limitación para la evacuación rectal de larga evolución, características valiosas en el caso de una intususcepción rectal.

El término úlcera rectal solitaria no es el más apropiado para este síndrome, ya que las úlceras se encuentran solo en el $40 \%$ de los pacientes. Alrededor del $20 \%$ tiene úlceras solitarias y el resto de las lesiones varían en forma y tamaño, y pueden involucrar desde lesiones de mucosa hiperémica hasta una lesión polipoide de base amplia (3).

La patogenia no está completamente dilucidada, pero hay varios factores que pueden contribuir a su desarrollo. Las diferentes teorías planteadas se podrían resumir en 4 (4):

1. el esfuerzo prolongado durante las deposiciones en el paciente que sufre de estreñimiento crónico puede provocar un trauma directo en la mucosa;

2. el trauma autoinducido por la instrumentación que ocurre cuando las personas intentan eliminar las heces impactadas por digitación rectal;

3. la contracción paradójica o no coordinada del músculo puborrectal asociada con el aumento de la presión intrarrectal, que resulta en isquemia y ulceración;

4. el prolapso rectal e invaginación intestinal que pueden conducir a trauma vascular localizado.

El síndrome también se superpone con trastornos de ansiedad y el trastorno obsesivo compulsivo (4).

Las manifestaciones clínicas son variables y pueden estar ausentes hasta en el $26 \%$ de los pacientes (5). Algunos síntomas incluyen sangrado rectal, secreción mucoide copiosa, esfuerzo excesivo prolongado para la defecación, dolor perineal y abdominal, sensación de defecación incompleta, estreñimiento $y$, raramente, prolapso rectal $(6,7)$. Respecto a los hallazgos endoscópicos, estos pueden variar desde eritema mucoso hasta úlceras simples o múltiples y lesiones polipoides. En la mayoría de los pacientes, las lesiones se localizan en la pared rectal anterior dentro de $10 s 10 \mathrm{~cm}$ del borde anal, pero también se pueden ubicar en el canal anal o en el colon sigmoide.

Las características histológicas incluyen obliteración fibromuscular de la lámina propia, extensión de fibras musculares e hipertrofia de la capa mucosa con alteración de la arquitectura de la cripta glandular. Hay otros cambios microscópicos menores como criptas distorsionadas y atipia epitelial reactiva, lo que puede conducir a diagnósticos erróneos, dado que los hallazgos similares pueden verse en EII y neoplasias. Sin embargo, la disposición difusa del colágeno en la lámina propia y las extensiones anormales de la fibra del músculo liso son marcadores sensibles para 
diferenciar el síndrome de úlcera rectal solitaria de otras afecciones a nivel histológico (3).

La evaluación del paciente con síndrome de úlcera rectal solitaria se puede complementar con el fin de evaluar la existencia de trastornos funcionales o prolapso rectal, lo que posibilita un mejor enfoque terapéutico. De esta manera, hay la posibilidad de realizar pruebas como la defecografía y el ultrasonido endoscópico. La defecografía permite visualizar diferentes etapas de la defecación, que determinan la presencia de invaginación intestinal o prolapso de la mucosa. Puede demostrar un prolapso oculto, así como alteración en la relajación del músculo puborrectal y un vaciamiento rectal incompleto o tardío. El ultrasonido endoscópico es una alternativa a la defecografía que permite evaluar el grosor del esfínter anal interno, así como la invaginación intestinal (8).

El manejo del síndrome de úlcera rectal solitaria depende de la gravedad de los síntomas y la presencia de prolapso rectal. En los pacientes asintomáticos, la observación y la educación son el pilar del abordaje. En los casos sintomáticos no graves, el tratamiento se basa en el uso de laxantes formadores de bolo y la terapia de biorretroalimentación, con el fin de corregir el comportamiento anormal del piso pélvico. Se reserva la cirugía para pacientes con prolapso rectal y para pacientes con síntomas graves que no responden al tratamiento conservador (9). Las opciones quirúrgicas incluyen escisión local, rectopexia o derivación fecal.
Se han descrito otros tipos de tratamientos que, aunque no tienen la suficiente evidencia, impactan en el manejo sintomático de los pacientes. Estos incluyen los tratamientos tópicos con sucralfato, salicilato, corticosteroides, sulfasalazina y mesalazina (10).

Dentro del diagnóstico diferencial del síndrome de úlcera rectal solitaria se incluyen la EII, colitis isquémica, proctitis infecciosa y neoplasias malignas. El pronóstico es difícil de determinar, ya que se trata de una afección crónica, con un importante impacto en la calidad de vida de las personas. Muchos de ellos persistirán con síntomas de disfunción anorrectal aún después del adecuado abordaje terapéutico.

\section{CONCLUSIÓN}

El síndrome se caracteriza por una defecación dolorosa y difícil, una sensación de evacuación incompleta y, a veces, hemorragia digestiva baja. Su presentación es variable, se presenta con baja incidencia y el diagnostico deberá ser integral con estudios endoscópicos, imágenes de radiología y confirmación histológica, considerando los diagnósticos diferenciales.

El abordaje multidisciplinario con el servicio de coloproctología y patología, así como la ampliación de la historia clínica asociada con los resultados de la defecografía y la revisión de literatura fueron los que brindaron un diagnóstico y manejo adecuados. Como resultado del abordaje diagnóstico de este caso, consideramos importante reconocer y reportar la existencia de este síndrome.

\section{REFERENCIAS}

1. Cruveihier J. Ulcer chronique du rectum. En: Bailliere JB. Anatomie pathologique du crops humain. París: 1829.

2. Madigan MR, Morson BC. Solitary ulcer of the rectum. Gut. 1969;10(11):871-81. https://doi.org/10.1136/gut.10.11.871

3. Zhu QC, Shen RR, Qin HL, Wang Y. Solitary rectal ulcer syndrome: clinical features, pathophysiology, diagnosis and treatment strategies. World J Gastroenterol. 2014;20(3):738-44. https://doi.org/10.3748/wjg.v20.i3.738

4. Forootan M, Darvishi M. Solitary rectal ulcer syndrome: A systematic review. Medicine (Baltimore). 2018;97(18):e0565. https://doi.org/10.1097/MD.0000000000010565

5. Ong J, Lim KH, Lim JF, Eu KW. Solitary caecal ulcer syndrome: our experience with this benign condition. Colorectal Dis. 2011;13(7):786-90. https://doi.org/10.1111/j.1463-1318.2010.02261.x

6. Kang YS, Kamm MA, Engel AF, Talbot IC. Pathology of the rectal wall in solitary rectal ulcer syndrome and complete rectal prolapse. Gut. 1996;38(4):587-90. https://doi.org/10.1136/gut.38.4.587
7. AlGhulayqah AI, Abu-Farhaneh EH, AlSohaibani FI, Almadi MA, AlMana HM. Solitary rectal ulcer syndrome: A single-center case series. Saudi J Gastroenterol. 2016;22(6):456-460. https://doi.org/10.4103/1319-3767.195555

8. Gopal DV, Young C, Katon RM. Solitary rectal ulcer syndrome presenting with rectal prolapse, severe mucorrhea and eroded polypoid hyperplasia: case report and review of the literature. Can J Gastroenterol. 2001;15(7):479-83. https://doi.org/10.1155/2001/145041

9. Torres C, Khaikin M, Bracho J, Luo CH, Weiss EG, Sands DR, et al. Solitary rectal ulcer syndrome: clinical findings, surgical treatment, and outcomes. Int J Colorectal Dis. 2007;22(11):1389-93. https://doi.org/10.1007/s00384-007-0344-5

10. Keshtgar AS, Ward HC, Sanei A, Clayden GS. Botulinum toxin, a new treatment modality for chronic idiopathic constipation in children: long-term follow-up of a doubleblind randomized trial. J Pediatr Surg. 2007;42(4):672-80. https://doi.org/10.1016/j.jpedsurg.2006.12.045 